\title{
Troponin I Biomarker for Cardiac Affection in Patients with Androgentic Aloepcia
}

A.A.Ibrahim, D.M.Elhabak, S.I.Farag, R.A.khashaba and D.F.Mohammed

Dermatology, Venerology and Andrology Dept., Faculty of Medicine, Benha Univ., Benha, Egypt

E-Mail:Shymaa@Doaa.com

\begin{abstract}
The plasma level of two common cardiovascular disease markers, cardiac troponin I and C-reactive protein, in alopecia areata and AGA affected subjects. We aimed to assesment of troponin I level of patients with AGA to detect if there is arole of troponin I as a cardiac biomarker for cardiac affection in the disease (AGA) pathogenesis and it's associations and looking for a hidden cardiac problem. The study was conducted on 75 participants; 50 patients suffering from Androgenetic alopecia, in addition to 25 control normal healthy persons. Norwood Classification for male Pattern Baldness Ludwig Classification of Female Pattern Baldness, ECG, Determination of serum Cardiac troponin I and Echocardiography were done for all participants. troponin I showed significant positive correlation with age, GLS, grades, as well as significant negative correlation with EF troponin I showed significant positive correlation with age, GLS, grades, as well as significant negative correlation with EF.
\end{abstract}

\section{Introduction}

Androgenetic alopecia (AGA) is aspecific sort of balding interceded by foundational androgens and hereditary variables. it is the most widely recognized kind of going bald in both genders [1].

Epidemiologic information shows that $80 \%$ of Caucasian men and 40-50\% of Caucasian ladies are influenced by AGA throughout their life, with predominance expanding with age. Predominance in the Asian populace is lower: AGA was seen in $14.1 \%$ of Korean men at all ages. Essentially, in the African populace, an examination uncovered that AGA commonness was discovered to be $14.6 \%$ in men and $3.5 \%$ in ladies [2].

Androgenetic alopecia is portrayed by reformist going bald, transcendently of the focal scalp, with some variety of designed misfortune. In spite of the fact that the commonness is high in old patients, AGA may likewise begin at pubescence. Patients who have unmistakable diminishing of hair are seen as more seasoned, which influences confidence and prompts psychosocial dismalness [3].

Androgenetic alopecia is portrayed by a stamped decline in hair follicle size, which could be identified with loss of hair follicle stem or begetter cell [4].

Androgenetic alopecia influences the two people. In men it produces male example going bald with bitemporal downturn and vertex hairlessness. In ladies it produces female example going bald (FPHL) with diffuse alopecia over the midfrontal scalp. FPHL happens because of nonuniform hair follicle scaling down inside follicular units [5].

The basic trichoscopic discoveries were hair breadth variety (HDD), structureless red regions (SRA), earthy colored spots (BD), and perifollicular white scales (PWS). Among the more uncommon trichoscopic discoveries were white specks (WD), yellow dabs (YD), multihair follicular unit (MHFU) and concealed hair (5)

Two medications are currently perceived as viable against AGA: skin minoxidil answer (for ladies and men) and finasteride tablets, $1 \mathrm{mg}$ (for men as it were). Autologous hair transplantation is a valuable treatment for cutting edge androgenetic alopecia [6].
Troponin complex is a segment of skeletal and heart muscle slight fibers. It comprises of three subunits: troponin I, T, and C, and it assumes an essential part in muscle action, associating changes in intracellular $\mathrm{Ca} 2+$ focus with age of constriction [7].

The plasma level of two regular cardiovascular infection markers, heart troponin I and C-receptive protein, in alopecia areata and AGA influenced subjects. The mean plasma cardiovascular troponin I level was most noteworthy in alopecia areata subjects, tolerably higher in AGA subjects, and least in subjects without going bald. The outcomes propose the potential for expanded heart rebuilding. Close observing of cardiovascular wellbeing in alopecia areata subjects, just as subsets of AGA patients, might be proper [8].

There is proof of subclinical perimyocardial disability in patients with SLE and liberated from cardiovascular indications. We further show that troponin I planning may can possibly distinguish subclinical myocardial contribution in patients with SLE [9].

We planned to assesment of troponin I level of patients with AGA to recognize if there is arole of troponin I as a heart biomarker for cardiovascular love in the sickness (AGA) pathogenesis and it's affiliations and searching for a shrouded cardiovascular issue.

\section{Patient and method}

This is a case-control study. The investigation was affirmed by the Local Ethics Committee on Research including human subjects of Benha Faculty of Medicine. Educated assents were acquired from all members before test assortment. The investigation was finished during the period from March 2019 to October 2019.

It was led in the Dermatology outpatient center, Faculty of Medicine, Benha University Hospital and Quisna focal emergency clinic. The investigation included 75 members; 50 patients experiencing Androgenetic alopecia, notwithstanding 25control typical solid people (male and female), age and sex coordinated people as a benchmark group.

Subjects were classified into 2 groups: 2.1.AGA group: This group included 50 patients with Androgenetic alopecia.Control group: This group 
included 25 apparently normalhealthy people age, sex and matched individuals. The extent of hair loss in male AGA affected subjects was classified using the Norwood scale, while female AGA subjects wereclassified using the Ludwig scale.

\subsection{Inclusion criteria}

Patients with androgenetic alopecia of both sexes' males and females. And age of onset of hair loss (12 to 49 years) with mean age of onset of hair loss 28.1 years.

\subsection{Exclusion criteria}

- Alopecia areata

- Scarring alopecia

- Trichotillomania

- Alopecia totalis

- Frontal fibrosing alopecia

- Patients on topical therapy (2weeks)

- Patients on systemic therapy (1month).

Norwood Classification for male Pattern Baldness Ludwig Classification of Female Pattern Baldness were done.

Full history taking, Complete general examination, Complete dermatological examination, Complete cardiac examination, ECG, Determination of serum Cardiac troponin I and Echocardiography were done for all participants.

\section{Results}

The AGA group mean age was 34.4 years, they were 31 males $(62 \%)$ and 19 females (38\%). In addition to 25 healthy control group, their mean age was 33 years, they were 11 males (44\%) and 14 females (56\%). Cases and control groups had matched age and gender ( $p>0.05$ for each).

Mean BMI for AGA group was $24.4 \mathrm{~kg} / \mathrm{m} 2$. Control group had matched BMI ( $>>0.05)$.

Although AGA group had significantly higher proportion of smokers when compared to control groups (30\% versus $16 \%$ ), there was no significant difference between both groups ( $\mathrm{p}>0.05)$.

Control group had no family history of chest pain, while cases had 6 FHOCP, with no significant difference between both groups (12\% versus $0 \%, \mathrm{p}>0.05)$.

AGA group had significantly lower EF when compared to control groups (mean $=58.4$ versus 61.1, $\mathrm{p}=0.005$ ).

AGA group had significantly lower absolute value of GLS when compared to control groups (mean $=-18.8$ versus $-20.7, \mathrm{p}<0.001)$.

Mean disease duration was 6.8 months. Disease onset was early in $72 \%$, late in $28 \%$, disease course was stationary in $36 \%$, progressive in $64 \%$.

Grades were assessed in all studied cases. Norwood grade system was used for males, 4 had grade II, 5 had grade III, 6 had grade IV, 8 had grade V, 3 had grade VI and 5 had grade VII. In addition, Ludwig grade system was used for females, 8 had grade I, 7 had grade II, 2 had grade III, 1 had grade IV and 1 had grade VI.

AGA group showed significantly higher Troponin I when compared to control group (mean=2.8 versus 1.6 , $\mathrm{p}<0.001)$.

Receiver operating characteristic (ROC) curve of EF, GLS and Troponin I levels was conducted for discrimination between AGA cases and control groups.

EF showed poor AUC (AUC=0.696). At cut off value of 60.5 , sensitivity was $76 \%$, specificity was $56 \%$, PPV was $77.6 \%$, NPV was $53.8 \%$, and accuracy was $69.3 \%$.

GLS showed good AUC (AUC=0.832). At cut off value of -19.5 , sensitivity was $70 \%$, specificity was $92 \%$, PPV was $94.6 \%$, NPV was $60.5 \%$, and accuracy was $77.3 \%$.

Troponin I showed excellent AUC (AUC=0.920). At cut off value of 1.95 , sensitivity was $78 \%$, specificity was $96 \%$, PPV was $97.5 \%$, NPV was $68.6 \%$, and accuracy was $84 \%$.

Troponin had better AUC than GLS which had better AUV than EF, for discrimination between AGA cases and control subjects.

Troponin I level increased significantly with male gender, early onset, higher grade in males and females. Otherwise, no significant associations were found regarding Troponin I level according to smoking, FH course in AGA group.

Troponin I showed significant positive correlation with age, GLS, grades, as well as significant negative correlation with EF, but not with BMI and duration in AGA group.

Straight relapse examination was led for forecast of lower EF inside contemplated subjects utilizing age, sex, BMI, smoking, beginning, course, term, presence of AGA as covariates. More established age, male sexual orientation, beginning stage, reformist course and presence of AGA were related with lower EF in Univariable examination. Nonetheless, taking huge covariates in univariable investigation into multivariable examination uncovered that more established age, male sex, beginning stage, and presence of AGA were considered as free danger indicators for lower EF.

Straight relapse investigation was directed for forecast of strange GLS inside contemplated subjects utilizing age, sexual orientation, BMI, smoking, beginning, course, span, presence of AGA as covariates. More established age, male sexual orientation, beginning stage, and presence of AGA were considered as autonomous danger indicator for strange GLS in uni-and multivariable examinations.

Straight relapse investigation was led for expectation of variables influencing troponin I level utilizing age, sex, BMI, smoking, beginning, course, term, presence of AGA as covariates. More seasoned age, male sex, beginning stage, and presence of AGA were considered as autonomous danger indicator for higher troponin $\mathrm{I}$ in uni-and multivariable investigations.

Strategic relapse investigation was directed for forecast of AGA advancement utilizing age, sexual orientation, BMI, smoking, EF, GLS and troponin I level as confounders. Lower EF, higher GLS and Troponin I level were altogether connected with AGA in univaiable investigation. Anyway utilizing critical confounders in 
univaiable investigation into multivariable examination uncovered that troponin I was considered as free danger indicator for AGA improvement.

Direct relapse investigation was led for forecast of AGA grades utilizing age, sex, BMI, smoking, beginning, course, span, EF, GLS and troponin I level as

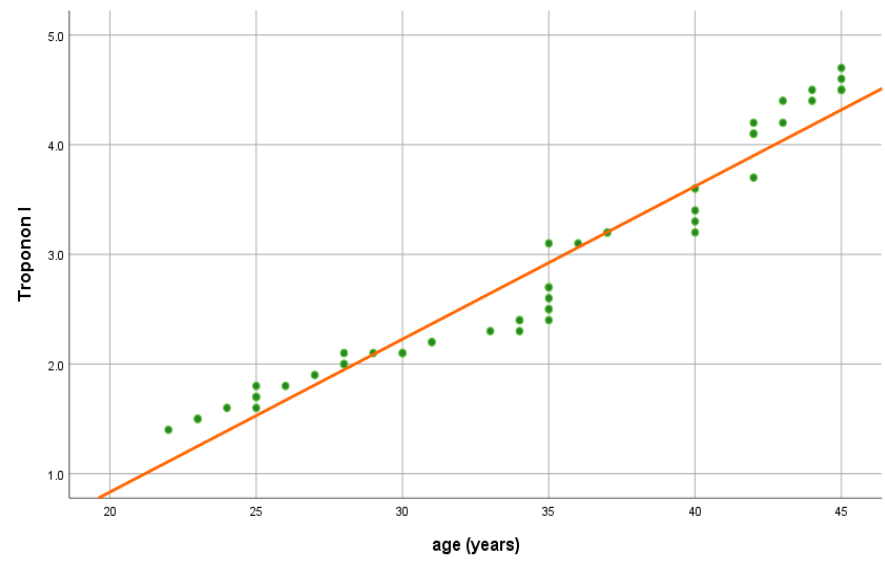

Fig (1) Correlations of Troponin I with age in AGA group.

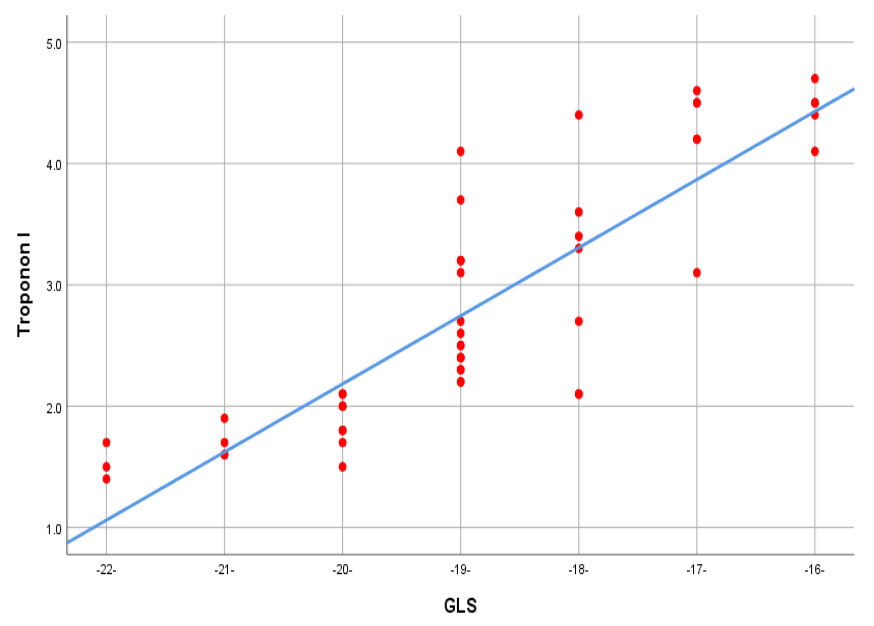

Fig (3) Correlations of Troponin I with GLS in AGA group.

\section{Discussion}

The current examination, we found that AGA gathering and control bunch had coordinated age, sexual orientation and BMI ( $\mathrm{p}>0.05)$.

The relationship among AGA and different CVDs have been proposed by various epidemiological examinations and meta-investigations. A few epidemiological examinations have announced a possible connection between balding and CVDs in guys. these examinations gave important first traces of possible affiliation. More controlled examinations with bigger companions suggested relationship between early AGA beginning and CVDs, particularly in guys with Type III vertex going bald [10].

Youthful time of AGA beginning is known to be related with expanded danger of CVDs sometime down the road. [8], demonstrated expanded plasma cTnI was commonly seen in more youthful male populaces with covariates. More seasoned age, male sexual orientation, lower EF, higher GLS and troponin I were related with higher AGA grades in univariable investigation. Utilizing these covariates in multivariable examination, just male sex and troponin I were considered as autonomous danger indicators for higher AGA grades.

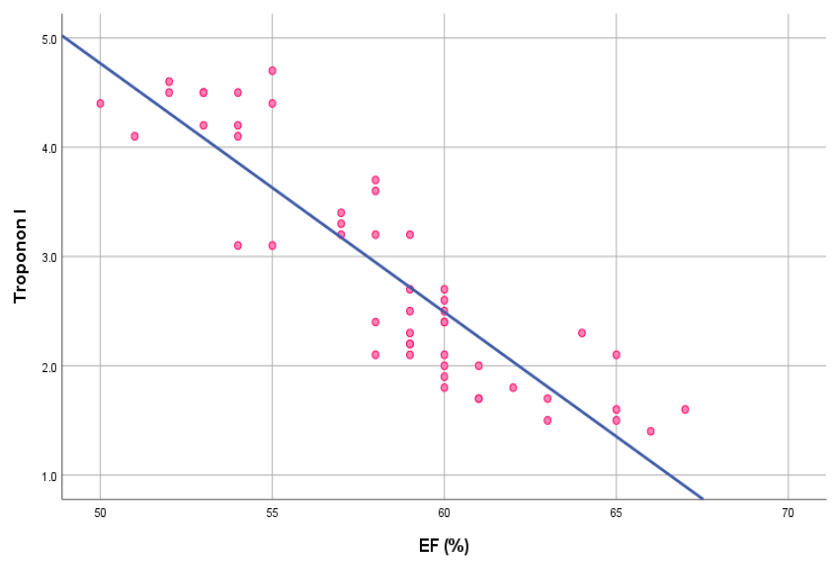

Fig (2) Correlations of Troponin I with EF in AGA group.

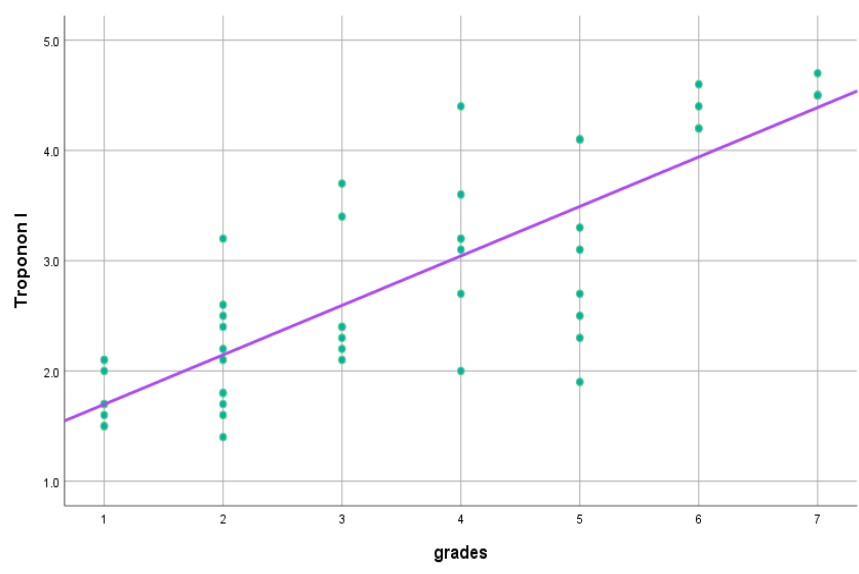

Fig (4) Correlations of Troponin I with grades in AGA group.

either AA or AGA ; furthermore, male AA patients communicated altogether higher measures of cTnI than AGA and non balding (NHL) guys. Given the clear connection between CVD danger and youthful age at AGA or AA beginning in guys [8].

Viable with our discoveries, [11] demonstrated that there was no factually noteworthy contrast in mean BMI esteems between patients with and without AGA in the two sexes. This was in accordance with [12] didn't discover any connection between's age, BMI and pervasiveness or seriousness of AGA in the two sexes.

Nonetheless, [12], [14] found that there were measurably huge contrasts with respect to weight list among cases and controls. Additionally, [15] study demonstrated that men with extreme sparseness had a higher mean weight record than men with lesser degrees of hair loss.

Stomach fat tissue is related with genuine metabolic 
problems, for example, IR, hyperinsulinemia, hypertension, expanded TGs, glucose narrow mindedness, and diabetes mellitus. A few examinations have highlighted stomach fat tissue and midriff perimeter as free danger factors for coronary illness (CHD) [13].

Insulin obstruction assumes a pathogenetic function in the scaling down of hair follicles. Vasoactive substances related with endothelial brokenness in IR lead to microcirculatory aggravation, perifollicular vasoconstriction, and multiplication of smooth muscle cells in the vascular divider. This condition prompts microvascular inadequacy, neighborhood tissue hypoxia, and reformist scaling down of hair follicles [16].

Hyperinsulinemia assume a function in nearby androgen creation, regardless of whether all over again from cholesterol or by locally changing over testosterone to DHT [17]. DHT hinders adenyl cyclase action, and can abridge the anagen cycle and could be answerable for the scaling down of follicles in AGA [18].

In the current investigation, Linear relapse examination was led for expectation of AGA grades utilizing age, sex, BMI, smoking, beginning, course, length, EF, GLS and troponin I level as covariates and we found that more seasoned age, male sex, lower EF, higher GLS and troponin I were related with higher AGA reviews in uni-variable examination.

These outcomes were in accordance with [14] which found that the smoking rate, midriff outline indicated an essentially expanding pattern as AGA was more serious. Additionally, a report by [19] was the first to show that the danger of CVD expanded in male AGA patients.

In an investigation directed by [13], the danger of CVD was higher with AGA of evaluation 3 vertex or more on the Norwood-Hamilton order scale, and especially the cardiovascular danger was essentially higher in the beginning stage AGA gathering.

Despite the fact that the [12] Study didn't discover hair loss to be related with an expanded danger of coronary illness, men younger than 55 years who had quick beginning and reformist AGA were discovered to be somewhat more inclined to creating coronary illness. Because of the different outcomes being accounted for by specialists, regardless of whether AGA expands the danger of CVD stays disputable.

All in all, we have exhibited a connection among's hairlessness and the danger of CVS ailments. This affiliation stayed huge recommending a typical yet denominator of these signs and CVS illnesses.

\section{References}

[1] S.Arias-Santiago, M.T.Gutiérrez-Salmerón, L.Castellote-Caballero. Androgenetic alopecia and cardiovascular risk factors in men and women: A comparative study. J Am Acad Dermatol,Vol.63, PP.420-9,2010.

[2] Y.Kelly, A.Blanco, A.Tosti. Androgenetic alopecia: an update of treatment options. Drugs.Vol. 76(14), PP.1349-1364,2016.

[3] M.Harries, A.Tosti, W.Bergfeld. Towards a consensus on how to diagnose and quantify female pattern hair loss - The 'Female Pattern Hair Loss
Severity Index (FPHL-SI)'. Journal Of European Academy Of Dermatology and Venerology (JEADV), Vol. 30(4), PP. 667-676,2015.

[4] H.S.Kamal, J.Anchal. Association between androgenetic alopecia and coronary artery disease in young male patients. International journal of trichology, Vol. 6, PP.(1),2014.

[5] R.Sinclair, N.Torkamani, L.Jones. New insights intothe pathogenesis and mechanism of hair loss. F1000Res.Vol.4 (F1000Faculty Rev), PP.585,2015.

[6] H.M.Almohanna, M.Perper, A.Tosti. Safety concerns when using novel medications to treat alopecia. Expert Opin Drug Saf, Vol.17(11), PP.1115-1128. ,2018.

[7] V.Vylegzhanina, A. E.Kogan, I. A.Katrukha. Anti-Cardiac Troponin Autoantibodies Are Specific to the Conformational Epitopes Formed by Cardiac Troponin I and Troponin $\mathrm{T}$ in the Ternary Troponin Complex. Clinical chemistry, Vol. 63(1), PP. 343-350,2017.

[8] E.H.C.Wang, L. L.Santos, Y.Xi. Alopecia Areata is Associated with Increased Expression of Heart Disease Biomarker $\mathrm{C}$ ardiac Troponin I Acta Dermato-Venereologica, Vol 98, Numbers 7-8, July, PP. 776-782(7), 2018.

[9] E.Colgecen, H.Ede, M.F.Erkoc, Akyuz. The relation of androgenetic alopecia severity with epicardial fat thickness. Annals of dermatology.Vol.28(2), PP. 205-209,2016.

[10] E.E.van der Wall. Male baldness; association with coronary artery disease?. Netherlands Heart Journal.Vol.23(5), PP. 263-264,2015.

[11] K.E.Salman, I.K.Altunay, N.A.Kucukunal. Frequency, severity and related factors of androgenetic alopecia in dermatology outpatient clinic: hospital-based cross-sectionalstudy inTurkey. An Bras Dermatol.Vol.2017, PP.92(1):35-40,2017.

[12] H.L.Su, L.S.Chen, S.C.Lin. Association of Androgenetic Alopecia With Mortality From Diabetes Mellitus and Heart Disease. JAMA Dermatology,Vol.149(5), PP.601. doi: 10. 1001/ jamadermatol.130,2013.

[13] V.Matilainen, M.Laakso, P.Hirsso. Hair loss, insulin resistance, and heredity in middleaged women. A population-based study. J Cardiovasc Risk, Vol.10, PP.227- 31. ,2003.

[14] S.Y. Park, S.S.Oh, W.S.Lee. Relationship between androgenetic alopecia and cardiovascular risk factors according to BASP classification in Koreans. The Journal of Dermatology.Vol.43(11), PP. 1293-1300. doi:10.1111/13468138.13355,2016.

[15] E.S.Ford, D.S.Freedman, T.Byers. Baldness andischemic heart disease in a national sample of men. Am J Epidemiol, Vol.143, PP.651-7,1996.

[16] O.Bakry, M.A.Shoeib, M.El Shafiee. Androgenetic alopecia, metabolic syndrome, and insulin resistance: Is there any association? A case-control study. Indian Dermatology Online Journal.Vol. 
5(3), 276. Doi, $\quad$ PP.10.4103/22295178.137776,2014.

[17] R.Horton, V.Pasupuletti, I.Antonipillai. Androgen induction of steroid 5a-reductase may be mediated via insulin-like growth factor 1. Endocrinology.Vol.133, PP.447-51,1993.

[18] J.Yip, M.B.Mattock, A.Morocutti. Insulin resistance in insulin-dependent diabetic patients with microalbuminuria. Lancet.Vol.342, PP.8837,1993 .

[19] S.G.Cotton, J.M.Nixon, R.G.Carpenter. Factors discriminating men with coronary heart disease from healthy controls. Br Heart J.Vol. 34, PP. 45864. , 1972 . 